\title{
Host Adaptation and Replication Properties of Two Bipartite Geminiviruses and Their Pseudorecombinants
}

\author{
Yu-Ming Hou, ${ }^{1}$ Epaminondas J. Paplomatas, ${ }^{2}$ and Robert L. Gilbertson ${ }^{1}$ \\ ${ }^{1}$ Department of Plant Pathology, University of California, Davis 95616 U.S.A.; ${ }^{2}$ Benaki Phytopathological \\ Institute, 8 S. Delta St., 14561 Kifissia, Athens, Greece \\ Accepted 2 December 1997
}

\begin{abstract}
To investigate factors involved in host adaptation and specificity of bipartite geminiviruses, the infectivity of bean dwarf mosaic (BDMV) and tomato mottle (ToMoV) geminiviruses and the BDMV/ToMoV pseudorecombinants [BDMV DNA-A + ToMoV DNA-B (BA+TB) and ToMoV DNA-A + BDMV DNA-B (TA+BB)] in Phaseolus vulgaris, Lycopersicon esculentum, Nicotiana benthamiana, and $N$. tabacum plants was determined. Additionally, replication of these viruses was examined in protoplasts prepared from $N$. tabacum BY2 and Xanthi-nc cells. In adapted hosts and the permissive experimental host, $N$. benthamiana, BDMV and ToMoV infected nearly $100 \%$ of inoculated plants, induced severe symptoms, and had high levels of both DNA components. In nonadapted hosts, BDMV and ToMoV infected approximately $40 \%$ of inoculated plants, induced no symptoms, and had reduced levels of both DNA components. For the pseudorecombinants, symptoms were observed only in $\mathrm{TA}+\mathrm{BB}$-infected $\boldsymbol{N}$. benthamiana and $P$. vulgaris plants. In the other pseudorecombinant/host combinations, symptomless infections were detected and some plants were infected with the DNA-A component only. Symptom development and/or higher infection rates for the pseudorecombinants were correlated with the host-adapted DNA-B component, and pseudorecombinant-infected plants had reduced levels of DNA-B. Protoplast replication assays revealed inefficient DNA-B replication for the pseudorecombinants, and differences in viral replication properties in the two $N$. tabacum cell lines.
\end{abstract}

Geminiviruses are a group of plant viruses characterized by circular, single-stranded DNA genomes and twinned icosahedral virions. These viruses have been classified into three subgroups based on insect vectors, host range, and genome organization (Faria et al. 1994; Rybicki 1994; Padidam et al. 1995). Subgroup III geminiviruses are transmitted by whiteflies (Bemisia tabaci Genn.) and usually have a bipartite genome composed of two approximately 2.6-kb DNA components, designated DNA-A and DNA-B (reviewed in Timmermans et al. 1994). The two

Corresponding author: Robert L. Gilbertson, Department of Plant Pathology, University of California, Davis 95616, U.S.A.; Phone: (530) 752-3163; Fax: (530) 752-5674; E-mail: rlgilbertson@ucdavis.edu
DNA components of a given geminivirus have little sequence similarity except for an approximately 200 nucleotide region, referred to as the common region (CR). The CR contains the viral origin of replication and virus-specific sequences that are recognized by the replication-associated (Rep or AC1) protein (reviewed in Arguello-Astorga et al. 1994; Laufs et al. 1995). The DNA-A component encodes for proteins involved in replication (Rep and AC3), gene expression (AC2), and encapsidation (AV1), whereas the DNA-B component encodes for two proteins (BV1 and BC1) involved in movement, host range, and symptom development.

Although the genes and genome organization of subgroup III geminiviruses are highly conserved, these viruses infect a wide range of plant species and often exhibit distinct host specificity. Since the characterization of the first subgroup III geminivirus in 1977, at least 58 geminiviruses infecting 40 different plant species have been described (Briddon and Markham 1995). Some crops, such as bean, tomato, and pepper, are infected by a diversity of subgroup III geminiviruses. Together, this suggests that subgroup III geminiviruses have the capacity for rapid evolution and host adaptation. Genetic analyses of the geminiviruses squash leaf curl (SqLCV) (Ingham and Lazarowitz 1993; Ingham et al. 1995) and African cassava mosaic (ACMV) (Haley et al. 1995) have shown that mutations in the $B V 1$ and $B C 1$ genes may restrict host specificity. However, using DNA-B hybrids made between the geminiviruses tomato golden mosaic (TGMV) and bean golden mosaic (BGMV), Schaffer et al. (1995) established that host specificity could not be solely attributed to DNA-Bencoded proteins. Furthermore, complementation studies revealed that the symptomless phenotype and reduced DNA levels of BGMV in the nonadapted host, Nicotiana benthamiana, could be attributed to functions associated with both DNA components (Petty et al. 1995). Stenger et al. (1992) showed that the capacity of TGMV to systemically infect different plant species also could be attributed to functions associated with both DNA components. Thus, host adaptation and specificity of bipartite geminiviruses may involve viral proteins and/or regulatory elements from both DNA components as well as host factors.

Bean dwarf mosaic (BDMV) and tomato mottle (ToMoV) are subgroup III geminiviruses isolated from common bean (Phaseolus vulgaris L.) in Colombia (Morales et al. 1990) and from tomato (Lycopersicon esculentum L.) in Florida 
(Abouzid et al. 1992; Gilbertson et al. 1993a), respectively. BDMV and ToMoV also infect $N$. benthamiana (Gilbertson et al. 1991a, 1993a), but induce different symptom phenotypes (Hou and Gilbertson 1996). Despite being identified from distinct plant species and geographic locations, BDMV and To$\mathrm{MoV}$ are closely related based on sequence similarity and the capacity to form infectious pseudorecombinants (Gilbertson et al. 1993a; Hou and Gilbertson 1996). Thus, it was hypothesized that BDMV and ToMoV evolved from a common ancestor and became adapted to P. vulgaris and L. esculentum, respectively (Gilbertson et al. 1993a). To test this hypothesis and to examine the role of the DNA-A and DNA-B components in host adaptation of these bipartite geminiviruses, the infectivity and replication properties of BDMV, ToMoV, and the BDMV/ToMoV pseudorecombinants [BDMV DNA-A + ToMoV DNA-B (BA+TB) and ToMoV DNA-A + BDMV DNA-B (TA+BB)] were determined in different plant species and in protoplasts, respectively. The results of this study revealed a significant contribution of the DNA-B component to host adaptation, inefficient DNA-B replication for the pseudorecombinants, and differences in viral replication properties in two N. tabacum cell lines.

\section{RESULTS}

Infectivity of BDMV, ToMoV, and the BA+TB and TA+BB pseudorecombinants in four plant species after delivery by agroinoculation.

Preliminary agroinoculation experiments established that stem puncture inoculation of $N$. benthamiana with BDMV and of $L$. esculentum and $N$. benthamiana with ToMoV provided approximately $100 \%$ infection, whereas leaf scratch inoculation of $P$. vulgaris with BDMV provided approximately $80 \%$ infection. In addition, symptoms in agroinoculated plants were indistinguishable from those induced by BDMV and ToMoV in these hosts after sap transmission (Gilbertson et al. 1993b). Therefore, agroinoculation was used to inoculate $P$. vulgaris, L. esculentum, $N$. benthamiana, and $N$. tabacum plants with BDMV, ToMoV, BA+TB, and TA+BB.
Ninety percent of $P$. vulgaris and $100 \%$ of $N$. benthamiana plants agroinoculated with BDMV developed severe leaf epinasty and distortion and stunted growth, whereas $100 \%$ of L. esculentum and $N$. benthamiana plants agroinoculated with ToMoV developed leaf epinasty and mottling (Table 1). Disease symptoms in BDMV-infected $N$. benthamiana plants appeared earlier and were more severe (more pronounced leaf epinasty and distortion and stunted growth) than symptoms in ToMoV-infected plants. L. esculentum and P. vulgaris plants agroinoculated with BDMV and ToMoV, respectively, did not develop symptoms. All N. tabacum plants agroinoculated with BDMV developed mild symptoms of leaf crumpling, mottling, and epinasty in newly emerged leaves, whereas plants agroinoculated with ToMoV did not develop symptoms. $\mathrm{TA}+\mathrm{BB}$ induced leaf mottling, crumpling, and epinasty in 92 and $100 \%$ of agroinoculated $P$. vulgaris and $N$. benthamiana plants, respectively (Table 1). No symptoms were observed for the other pseudorecombinant/host combinations, i.e., TA+ $\mathrm{BB}$ in L. esculentum and N. tabacum, and BA+TB in P. vulgaris, L. esculentum, N. benthamiana, and N. tabacum.

For virus/host combinations that failed to show obvious disease symptoms, newly emerged leaves from all inoculated plants were examined for infection by polymerase chain reaction (PCR) analysis with degenerate DNA-A and DNA-B primers. The results of these analyses for three independent experiments are presented in Table 1. N. tabacum plants agroinoculated with ToMoV or BA+TB were not systemically infected. Symptomless infections were detected in $38 \%$ of $L$. esculentum plants agroinoculated with BDMV and in $44 \%$ of $P$. vulgaris plants agroinoculated with ToMoV. Symptomless infections also were detected in $39 \%$ of $P$. vulgaris, $70 \%$ of $L$. esculentum, and $100 \%$ of $N$. benthamiana plants agroinoculated with BA+TB, and in $21 \%$ of L. esculentum and $43 \%$ of N. tabacum plants agroinoculated with TA+BB (Table 1).

Both DNA components were detected in all ToMoVinfected $P$. vulgaris, BA+TB-infected $N$. benthamiana, and TA+BB-infected N. tabacum plants (Table 1). However, for the other virus/host combinations in which symptomless infections were detected (primarily the pseudorecombinants),

Table 1. Infectivity of bean dwarf mosaic geminivirus (BDMV), tomato mottle geminivirus (ToMoV), BA+TB, TA+BB, or the DNA-A components of BDMV or ToMoV in agroinoculated Nicotiana tabacum, $N$. benthamiana, Phaseolus vulgaris, and Lycopersicon esculentum plants, and detection of geminivirus DNA-A and DNA-B components by polymerase chain reaction (PCR) analysis ${ }^{\mathrm{a}}$

\begin{tabular}{|c|c|c|c|c|c|c|c|c|}
\hline \multirow[b]{2}{*}{ Inoculum } & \multicolumn{4}{|c|}{ Detection of DNA-A } & \multicolumn{4}{|c|}{ Detection of DNA-B } \\
\hline & N. tabacum & N. benthamiana & P. vulgaris & L. esculentum & N. tabacum & N. benthamiana & P. vulgaris & L. esculentum \\
\hline \multirow[t]{2}{*}{$\mathrm{BA}+\mathrm{TB}$} & $0 / 7^{\mathrm{b}}$ & $9 / 9$ & $11 / 28$ & $21 / 30$ & $0 / 7$ & $9 / 9$ & $3 / 28$ & $4 / 30$ \\
\hline & $0 \%$ & $100 \%$ & $39 \%$ & $70 \%$ & $0 \%$ & $100 \%$ & $11 \%$ & $13 \%$ \\
\hline \multirow[t]{2}{*}{$\mathrm{TA}+\mathrm{BB}$} & $3 / 7$ & $9 / 9^{*}$ & $24 / 26^{*}$ & $6 / 29$ & $3 / 7$ & $9 / 9 *$ & $24 / 26^{*}$ & $0 / 29$ \\
\hline & $43 \%$ & $100 \%$ & $92 \%$ & $21 \%$ & $43 \%$ & $100 \%$ & $92 \%$ & $0 \%$ \\
\hline \multirow[t]{2}{*}{ BDMV } & $6 / 6^{* c}$ & $7 / 7 *$ & $19 / 21 *$ & $11 / 29$ & $6 / 6^{*}$ & $7 / 7^{*}$ & $19 / 21 *$ & $9 / 29$ \\
\hline & $100 \%$ & $100 \%$ & $90 \%$ & $38 \%$ & $100 \%$ & $100 \%$ & $90 \%$ & $31 \%$ \\
\hline \multirow[t]{2}{*}{ ToMoV } & $0 / 7$ & $8 / 8^{*}$ & $12 / 27$ & $24 / 24 *$ & $0 / 7$ & $8 / 8^{*}$ & $12 / 27$ & $24 / 24 *$ \\
\hline & $0 \%$ & $100 \%$ & $44 \%$ & $100 \%$ & $0 \%$ & $100 \%$ & $44 \%$ & $100 \%$ \\
\hline \multirow[t]{2}{*}{ BDMV-A } & $\mathrm{ND}^{\mathrm{d}}$ & $5 / 5$ & $2 / 10$ & $3 / 9$ & ND & $0 / 5$ & $0 / 10$ & $0 / 9$ \\
\hline & & $100 \%$ & $20 \%$ & $33 \%$ & & $0 \%$ & $0 \%$ & $0 \%$ \\
\hline \multirow[t]{2}{*}{ ToMoV-A } & ND & $4 / 4$ & $0 / 9$ & $4 / 10$ & ND & $0 / 4$ & $0 / 9$ & $0 / 10$ \\
\hline & & $100 \%$ & $0 \%$ & $40 \%$ & & $0 \%$ & $0 \%$ & $0 \%$ \\
\hline
\end{tabular}

${ }^{a}$ Data represent totals for three independent experiments except for BDMV-A and ToMoV-A, which represent totals for two independent experiments.

${ }^{b}$ Number of infected plants (i.e., the presence of disease symptoms or detection of the appropriate DNA component by PCR analysis)/total number of plants inoculated. This is also presented as percentage of total plants infected.

${ }^{c}$ Asterisks indicate host/virus combinations in which disease symptoms were observed.

${ }^{\mathrm{d}}$ Not determined. 
the DNA-A component only was detected in some plants (Table 1). For example, DNA-A was detected in 11 of $28 P$. vulgaris plants agroinoculated with $\mathrm{BA}+\mathrm{TB}$, whereas DNA-B was detected in only 3 of these 11 plants. Similarly, DNA-A was detected in 21 of $30 \mathrm{~L}$. esculentum plants agroinoculated with BA+TB, but DNA-B was detected in only 4 of these 21 plants. DNA-A was detected in 6 of 29 L. esculentum plants agroinoculated with $\mathrm{TA}+\mathrm{BB}$, but DNA-B was not detected in any of these plants.

These infectivity results suggest that $P$. vulgaris and $L$. esculentum are adapted hosts for BDMV and ToMoV, respectively, and that $L$. esculentum and $P$. vulgaris are nonadapted hosts for BDMV and ToMoV, respectively. Furthermore, symptom development and/or higher infectivity rates for the pseudorecombinants were correlated with the host-adapted DNA-B component, whereas the capacity to systemically infect $N$. tabacum cv. Xanthi-nc plants was associated with the BDMV DNA-B component.

\section{Analyses of viral DNA levels in agroinoculated plants.}

Viral DNA levels in infected $N$. benthamiana, $P$. vulgaris, and L. esculentum plants were determined by Southern blot hybridization analysis with DNA-A-specific and DNA-B-specific probes. Both DNA components were detected in $N$. benthamiana plants infected with BDMV, ToMoV, and the pseudorecombinants. DNA-A levels were similar in plants infected with each of the viruses, and DNA-B levels in BDMV- and ToMoVinfected plants were similar to those detected for DNA-A (Fig. 1). However, DNA-B levels in BA+TB- and TA+BB-infected plants were only 42 and $34 \%$, respectively, of those detected for their respective DNA-A components (Fig. 1).

BDMV-infected $P$. vulgaris plants had high levels of DNAA and DNA-B, and levels of each component were similar (lanes 13 and 14 in Figure 2). These results further establish that $P$. vulgaris is an adapted host for BDMV. TA+BBinfected $P$. vulgaris plants had DNA-A levels that were $110 \%$ of those detected in BDMV-infected plants (Fig. 2), but DNA$\mathrm{B}$ levels in the TA+BB-infected plants were only $71 \%$ of those detected in BDMV-infected plants (Fig. 2). DNA-A and DNA-B were detected in ToMoV-infected $P$. vulgaris plants, but at reduced levels compared with those in BDMV- or TA+BB-infected plants (Fig. 2). Interestingly, DNA-A levels in ToMoV-infected $P$. vulgaris plants were lower than those detected for DNA-B (Fig. 2). For P. vulgaris plants agroinoculated with $\mathrm{BA}+\mathrm{TB}$, two plants were selected in which both DNA components were detected by PCR, and two other plants were selected in which DNA-A only was detected. DNA-A was detected in all four plants, but levels were much greater in plants in which DNA-B also was detected: in the absence of DNA-B, DNA-A was detected only after a 6-day exposure (data not shown); in the presence of DNA-B, DNA-A was detected after a 10-h exposure (Fig. 2). In addition, DNA-B levels in BA+TBinfected plants were reduced compared with those detected for DNA-A (lanes 1 and 2 in Figure 2).

ToMoV-infected L. esculentum plants had high levels of DNA-A and DNA-B, and levels of each component were similar (lanes 13 and 14 in Figure 3). These results further establish that $L$. esculentum is an adapted host for ToMoV. Three of four BDMV-infected L. esculentum plants had similar levels of DNA-A and DNA-B (lanes 9, 10, and 12 in Figure 3), and the DNA-A and DNA-B levels in these plants were
19 and $18 \%$, respectively, of those detected in ToMoVinfected plants. In the other BDMV-infected L. esculentum plant, DNA-A only was detected (lane 11 in Figure 3), and at a slightly reduced level compared with those detected in plants infected with BDMV DNA-A and DNA-B. Consistent with results of PCR analysis, DNA-A only was detected in TA+BB-infected L. esculentum plants (lanes 5 to 8 in Figure 3 ), and DNA-A levels in these plants were $14 \%$ of those detected in ToMoV-infected plants. DNA-A was detected in all BA+TB-infected plants, whereas DNA-B was detected in two of these plants and at reduced levels compared with those detected for DNA-A (lanes 2 and 3 in Figure 3). DNA-A and DNA-B levels in BA+TB-infected plants were 24 and $3 \%$, respectively, of those detected in ToMoV-infected plants.

These Southern blot hybridization results establish that BDMV DNA-A and ToMoV DNA-A can replicate in the nonadapted hosts $L$. esculentum and $P$. vulgaris, respectively. Furthermore, in N. benthamiana, P. vulgaris, and L. esculentum plants infected with the BDMV/ToMoV pseudorecombinants, DNA-B levels were reduced (or absent in the case of TA+BB-infected L. esculentum plants) compared with levels of DNA-A (Figs. 1, 2, and 3).

\section{Agroinoculation with BDMV or ToMoV DNA-A.}

Agroinoculation experiments with the pseudorecombinants suggested that BDMV DNA-A and ToMoV DNA-A could systemically infect $P$. vulgaris and $L$. esculentum plants in the absence of DNA-B. Although PCR and Southern blot hybridization analyses failed to reveal even trace amounts of DNA-B in these plants, this did not rule out an initial contribution by DNA-B. Therefore, N. benthamiana, P. vulgaris, and L. esculentum plants were agroinoculated with BDMV DNA-A or ToMoV DNA-A. In two independent experiments, none of the agroinoculated plants developed symptoms. However, BDMV

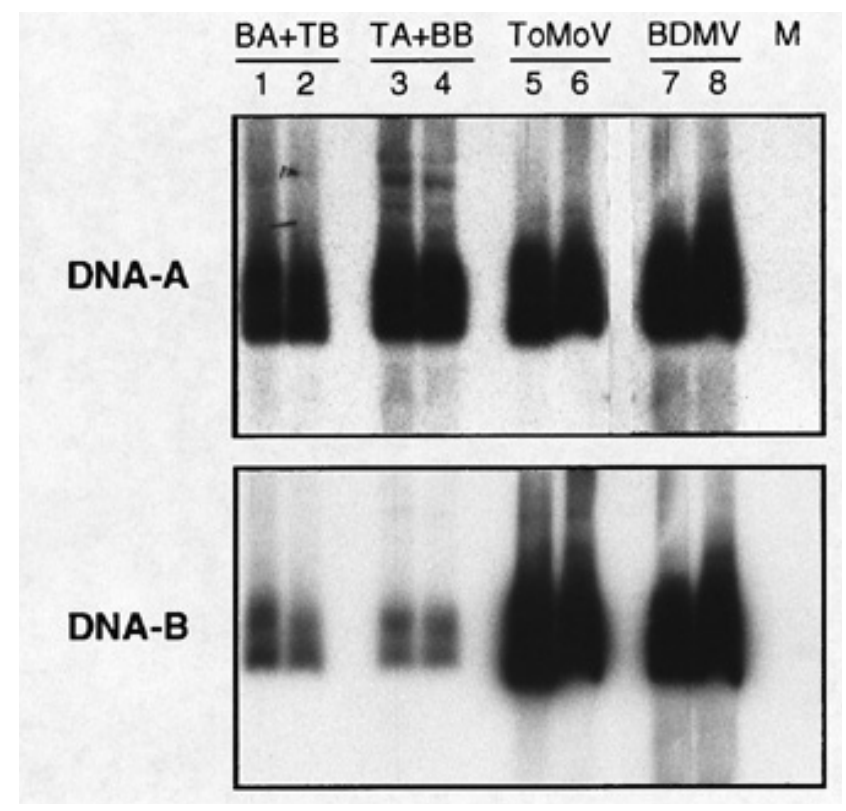

Fig. 1. Southern blot hybridization analysis of total nucleic acids extracted from Nicotiana benthamiana plants infected with BA+TB (lanes 1 and 2), TA+BB (lanes 3 and 4), ToMoV (lanes 5 and 6), and BDMV (lanes 7 and 8) via agroinoculation, and from a noninoculated plant (lane M). Blots were probed with specific probes for DNA-A or DNA-B. 
DNA-A and ToMoV DNA-A infections were detected by PCR in $100 \%$ of $N$. benthamiana, 20 and $0 \%$ of P. vulgaris, respectively, and 33 and $40 \%$ of L. esculentum plants, respectively (Table 1). DNA-B was not detected in any of these plants by PCR (Table 1) or by Southern blot hybridization analysis (data not shown).

Southern blot hybridization analyses revealed that DNA-A levels in BDMV DNA-A-infected and ToMoV DNA-Ainfected $N$. benthamiana plants were 28 and $22 \%$, respectively, of those detected in BDMV-infected $N$. benthamiana plants (data not shown). DNA-A levels in BDMV DNA-Ainfected $P$. vulgaris and L. esculentum plants were 5 and $8 \%$, respectively, of those detected in BDMV-infected $N$. benthamiana plants, whereas DNA-A levels in ToMoV DNA-Ainfected L. esculentum plants were $7 \%$ of those detected in BDMV-infected $N$. benthamiana plants (data not shown). Together, these results indicate that BDMV and ToMoV DNA-A have the capacity to systemically infect plants when delivered by agroinoculation, that the efficiency of these infections varies among plant species, and that DNA-A levels are considerably reduced in DNA-A-infected plants.

\section{Replication kinetics of viral DNA components} in $N$. tabacum protoplasts.

It has been hypothesized that reduced DNA-B levels in plants infected with the BDMV/ToMoV pseudorecombinants are due to inefficient DNA-B replication (Hou and Gilbertson 1996). To test this hypothesis, replication kinetics of the DNA components of BDMV, ToMoV, BA+TB, and TA+BB were examined in protoplasts generated from suspension cultures of two N. tabacum cell lines, BY2 and Xanthi-nc. Inocula were full-length monomers or 1.5-mers/dimers. Three independent experiments were conducted with each type of inoculum in each cell line, and viral replication was examined by Southern blot hybridization analysis with DNA-A-specific and DNAB-specific probes.

In BY2 cells, newly replicated single-stranded (ss) and double-stranded (ds) DNA-A and DNA-B forms (determined based upon treatment with methylation-sensitive enzymes and $\mathrm{S} 1$ nuclease, data not shown) were detected $48 \mathrm{~h}$ post electroporation (hpe), except for DNA-B of TA+BB, which was detected 72 hpe (Fig. 4). DNA-A and DNA-B levels increased throughout the duration of the experiment (120 hpe). DNA-A replication kinetics were similar for BDMV and BA+TB (i.e., BA+TB DNA-A levels were $99 \%$ of those detected for BDMV). However, overall replication of ToMoV and TA+BB DNA-A was reduced compared with that of BDMV and BA+TB DNA-A (i.e., ToMoV and TA+BB DNA-A levels were 40 and $56 \%$, respectively, of those detected for BDMV DNA-A) (Fig. 4). Thus, BDMV DNA-A replicated more efficiently than ToMoV DNA-A in this cell line. Replication kinetics of BDMV DNA-B and ToMoV DNA-B were similar to

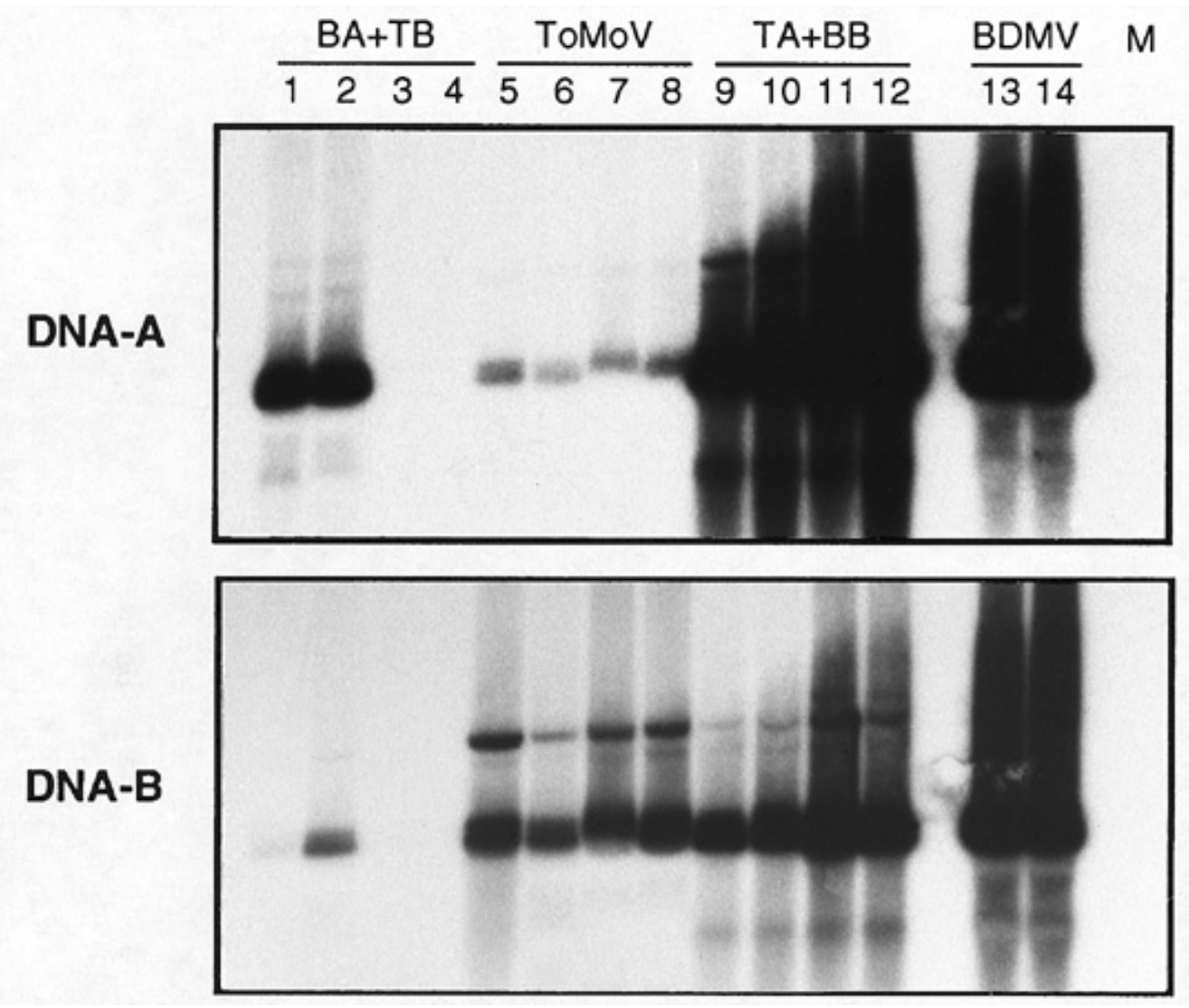

Fig. 2. Southern blot hybridization analysis of total nucleic acids extracted from Phaseolus vulgaris plants infected with BA+TB (lanes 1 to 4$)$, ToMoV (lanes 5 to 8), TA+BB (lanes 9 to 12), and BDMV (lanes 13 and 14) via agroinoculation, and from a noninoculated plant (lane M). Approximately 0.5 $\mu \mathrm{g}$ of total nucleic acids per plant was analyzed for plants with symptoms (TA+BB and BDMV), whereas approximately $2 \mu \mathrm{g}$ was analyzed for plants with symptomless infections (BA+TB and ToMoV). Blots were probed with specific probes for DNA-A or DNA-B. 
those of their respective DNA-A components (i.e., BDMV and ToMoV DNA-B levels were 95 and 94\%, respectively, of those detected for their DNA-A components) (Fig. 4). However, ToMoV DNA-B levels were only $40 \%$ of those detected for BDMV DNA-B, which presumably reflects the difference between ToMoV and BDMV DNA-A replication. For both pseudorecombinants, DNA-B replication was reduced compared with that of their respective DNA-A components (Fig. 4). This reduction was more pronounced for $\mathrm{TA}+\mathrm{BB}$ (i.e., $\mathrm{BA}+\mathrm{TB}$ and $\mathrm{TA}+\mathrm{BB}$ DNA-B levels were 68 and $15 \%$, respectively, of those detected for their DNA-A components).

Parallel experiments conducted with Xanthi-nc cells revealed differences in viral replication kinetics compared with BY2 cells. First, newly replicated ss and ds DNA forms were detected 24 hpe, except for the DNA-B components of $\mathrm{BA}+\mathrm{TB}$ and $\mathrm{TA}+\mathrm{BB}$, which were detected 48 hpe (Fig. 5). Second, overall DNA-A and DNA-B replication was greater, and more multimeric viral DNA forms were detected in Xanthi-nc cells (compare newly replicated DNA forms in Figures 4 and 5). Similar DNA-A replication kinetics were detected for all four viruses (i.e., DNA-A levels for BA+TB, ToMoV, and $\mathrm{TA}+\mathrm{BB}$ were 98,93 , and $92 \%$, respectively, of those detected for BDMV DNA-A) (Fig. 5). Thus, ToMoV DNA-A replicated more efficiently in Xanthi-nc cells than in BY2 cells (compare Figures 4 and 5). The replication kinetics of BDMV DNA-B were similar to those of BDMV DNA-A, whereas ToMoV DNA-B replication was reduced compared with that of ToMoV DNA-A (i.e., ToMoV DNA-B levels were $70 \%$ of those detected for ToMoV DNA-A). ToMoV DNA-B levels were $65 \%$ of those detected for BDMV DNA-B (Fig. 5) but, in contrast to the BY2 cell line, this could not be attributed to inefficient ToMoV DNA-A replication. For both pseudorecombinants, DNA-B replication was reduced compared with that of their respective DNA-A components (Fig. 5), and this reduction was greater than that observed for ToMoV DNA-B. In Xanthi-nc cells, the reduction in the DNA-B level was similar for both pseudorecombinants (i.e., BA+TB and TA+BB DNA-B levels were 58 and $62 \%$, respectively, of those detected for their DNA-A components) (Fig. 5).

Similar results were obtained in both cell lines with monomers and 1.5-mers/dimers. Furthermore, protoplast viability and electroporation efficiency (as determined by control experiments with a 35S-green fluorescent protein gene construct) were similar for these two cell lines. Thus, these results support the hypothesis that DNA-B replication is less efficient for the pseudorecombinants, and also reveal differences in viral replication properties in these two cell lines.

\section{DISCUSSION}

The hosts of a virus can be defined as plants in which the virus can replicate and spread systemically. Host plants can be divided into "natural hosts," which are infected with a virus in natural habitats and/or in agricultural situations, and "experimental hosts," which are generally infected with a virus under laboratory conditions (Dawson and Hilf 1992). Because viral evolution is an ongoing rather than a static process, natural hosts can be further subdivided into adapted hosts, in which a virus can efficiently replicate and induce symptoms (usually the host or hosts a virus most commonly infects in nature), and

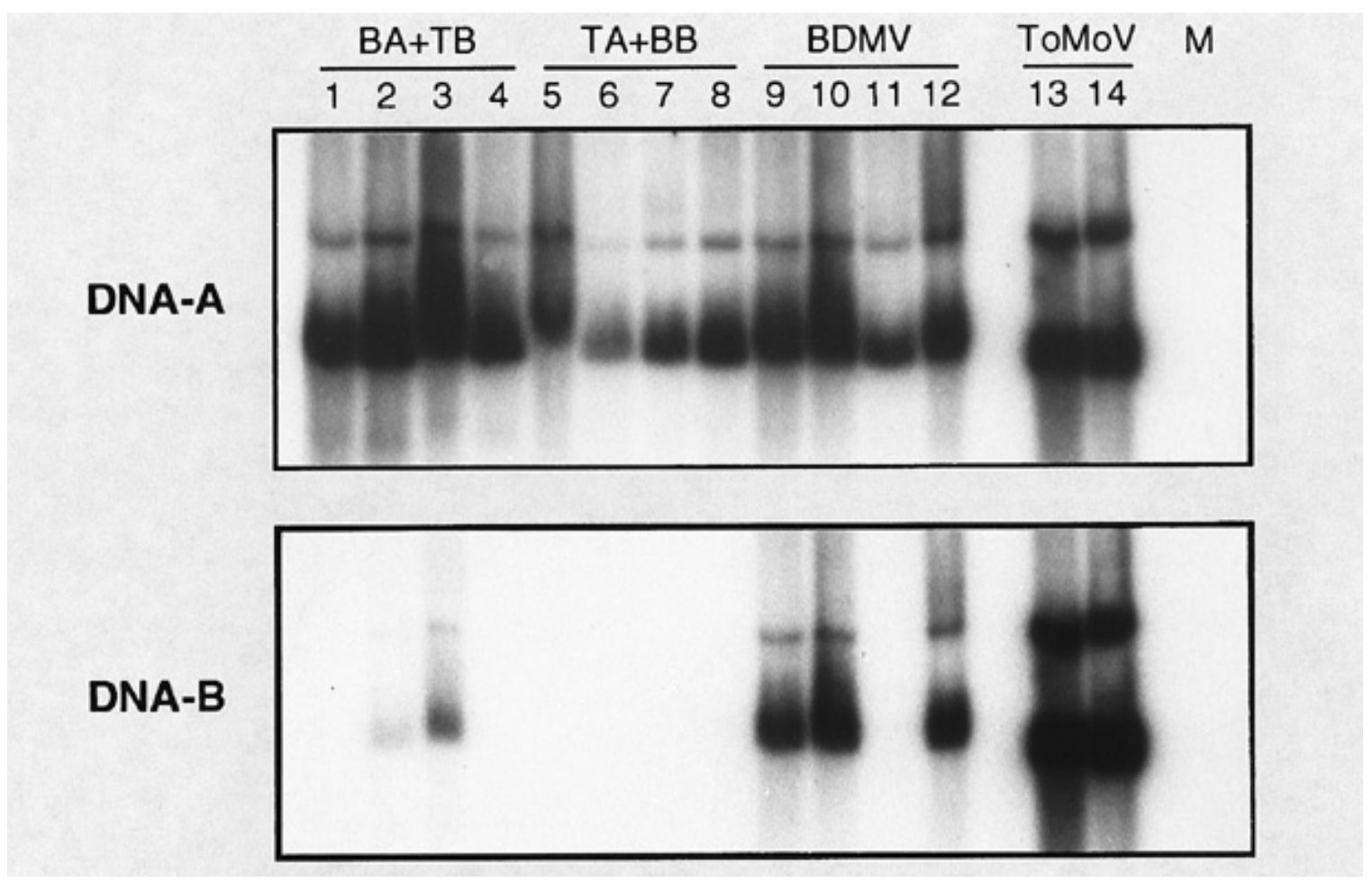

Fig. 3. Southern blot hybridization analysis of total nucleic acids extracted from Lycopersicon esculentum plants infected with BA+TB (lanes 1 to 4), $\mathrm{TA}+\mathrm{BB}$ (lanes 5 to 8), BDMV (lanes 9 to 12), and ToMoV (lanes 13 and 14) via agroinoculation, and from a noninoculated plant (lane M). Approximately $0.5 \mu \mathrm{g}$ of total nucleic acids per plant was analyzed for plants with symptoms (ToMoV), whereas approximately $2 \mu \mathrm{g}$ was analyzed for plants with symptomless infections (BA+TB, TA+BB, and BDMV). Blots were probed with specific probes for DNA-A or DNA-B. 
nonadapted hosts, which are poorly infected and may not be recognized as a host. Nonadapted hosts are often characterized by low infection rates and/or mild or no symptoms.

P. vulgaris and L. esculentum are adapted hosts for BDMV and ToMoV, respectively, based on high infection rates, symptomatic phenotypes, and high levels of both DNA components detected for these virus/host combinations. Additionally, the equivalent levels of DNA-A and DNA-B detected for these combinations are consistent with the concept that bipartite geminiviruses tend to maintain similar levels of both components in adapted hosts (Hou and Gilbertson 1996). Infection properties of both viruses in the experimental host $N$. benthamiana were similar to those in adapted hosts. However, this probably can be attributed to the permissive nature of $N$. benthamiana to viral infection (Dawson and Hilf 1992) rather than viral host adaptation. The infectivity results also indicate that, under the conditions of this study, $N$. tabacum cv. Xanthi-nc is a host for BDMV, but not for ToMoV. The high infection rate and the mild symptom phenotype for BDMV in these plants suggest that N. tabacum may be in between an adapted and nonadapted host for BDMV. The findings that BDMV was more pathogenic than ToMoV in $N$. benthamiana and $N$. tabacum, and that BDMV replicated to higher levels than ToMoV in N. tabacum cells demonstrate that a virus adapted to a leguminous host can infect certain solanaceous species more efficiently than a virus adapted to another solanaceous host.
Low infection rates, symptomless phenotypes, and reduced DNA levels were detected in $L$. esculentum and P. vulgaris plants agroinoculated with BDMV and ToMoV, respectively. These results establish that $L$. esculentum and $P$. vulgaris are nonadapted hosts rather than nonhosts for BDMV and To$\mathrm{MoV}$, respectively. Similar phenotypes have been described for other bipartite geminiviruses in nonadapted or experimental hosts, e.g., a restricted host range isolate of SqLCV in $P$. vulgaris (Lazarowitz 1991), BDMV and BGMV in Glycine max (Gilbertson et al. 1991a), TGMV in P. vulgaris (Petty et al. 1995), and BGMV in N. benthamiana (Petty et al. 1995). These phenotypes may represent the initial stages of adaptation to these host species, or changes (e.g., mutations) in a progenitor virus that allowed for efficient infection of one host species (adapted host) at the expense of efficient infection of other hosts (nonadapted hosts). The latter scenario could be the case for BDMV and ToMoV, which probably evolved from an Abutilon mosaic-like geminivirus infecting the ubiquitous weed, Sida spp. (Gilbertson et al. 1993a; Hofer et al. 1997). Independent introduction events of this "progenitor" geminivirus into $P$. vulgaris and $L$. esculentum plants, followed by repeated passage through these hosts mediated by whitefly transmission, may have given rise to BDMV and ToMoV, respectively. Moreover, the identification of nonadapted hosts reveals that host specificity of some bipartite geminiviruses is not as distinct as previously believed. The significance, if any, of nonadapted hosts in the

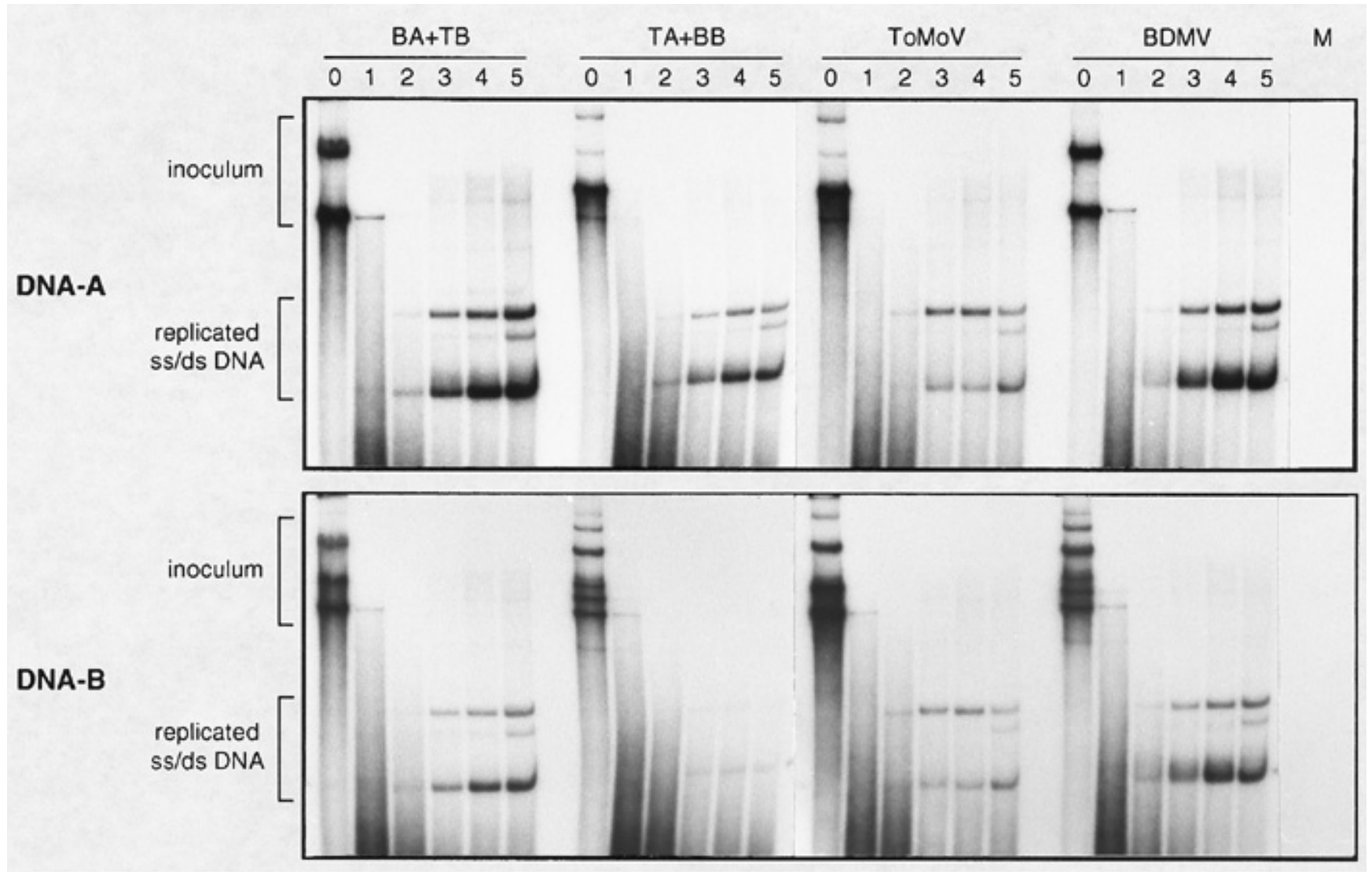

Fig. 4. Southern blot hybridization analysis of total nucleic acids extracted from Nicotiana tabacum BY2 cells transfected with BA+TB, TA+BB, To$\mathrm{MoV}$, and BDMV at 0,24, 48, 72, 96, and $120 \mathrm{~h}$ post electroporation, lanes 0 to 5, respectively; and nontransfected cells (lane M). Blots were probed with specific probes for DNA-A or DNA-B. Positions of input inoculum and newly replicated viral DNA are indicated with brackets. 
epidemiology of geminivirus diseases is unknown, but infections of nonadapted hosts could provide additional inoculum sources (particularly in the absence of adapted hosts), or result in mixed infections with other geminiviruses, which could facilitate viral evolution through pseudorecombination or recombination.

The symptomless phenotype of BDMV and ToMoV in nonadapted hosts is probably due to reduced levels of DNA-B and DNA-B-encoded gene products, because geminivirus symptom determinants have been mapped to DNA-B (von Arnim and Stanley 1992; Ingham et al. 1995; Pascal et al. 1993; Schaffer et al. 1995). The overall reduced DNA levels in nonadapted hosts likely reflect impaired viral movement due to inefficient expression and/or function of the BV1 and BC1 movement proteins (MPs) as suggested by Petty et al. (1995). Low infection rates in nonadapted hosts could also reflect inefficient cell-to-cell and/or long-distance movement, which may allow the host defense system to restrict virus movement (Mise and Ahlquist 1995) and/or prevent the virus from accessing critical cell types needed to establish and maintain a systemic infection, e.g., protophloem cells in the shoot apex (Wang et al. 1996).

With the exception of $P$. vulgaris and $N$. benthamiana plants infected with $\mathrm{TA}+\mathrm{BB}$, plants infected with the BDMV/ ToMoV pseudorecombinants did not exhibit obvious symp- toms. The pathogenicity and high rates of infection of $\mathrm{TA}+\mathrm{BB}$ in $P$. vulgaris likely reflect the efficient function and/or expression of the BDMV BV1 and BC1 MPs in the adapted host. Of particular interest was the failure of BA+TB to induce symptoms in L. esculentum, because this pseudorecombinant has the DNA-B component of the host-adapted virus. However, BA+TB was more infectious in L. esculentum plants than $\mathrm{TA}+\mathrm{BB}$, which is consistent with the more efficient expression and/or function of the ToMoV BV1 and BC1 MPs in the adapted host. The role of DNA-B in host adaptation of BDMV and ToMoV is further supported by the findings that a given DNA-A component was more infectious in a nonadapted host as a pseudorecombinant with the DNA-B component of the host-adapted virus than with its own nonhost-adapted DNA-B component, and that infectivity in $N$. tabacum cv. Xanthi-nc plants was associated with the BDMV DNA-B component. The DNA-A component also can be a determinant of host adaptation and/or specificity. For example, replication deficiencies have been implicated in the inability of Indian cassava mosaic geminivirus and TGMV to infect nonhost species (Frischmuth et al. 1993; Stenger et al. 1992), and of a restricted host range isolate of SqLCV to infect a nonadapted host (Lazarowitz 1991). A DNA-A replication deficiency was not found for TA+BB in P. vulgaris, but could not be ruled out in the case of $\mathrm{BA}+\mathrm{TB}$ in L. esculentum.

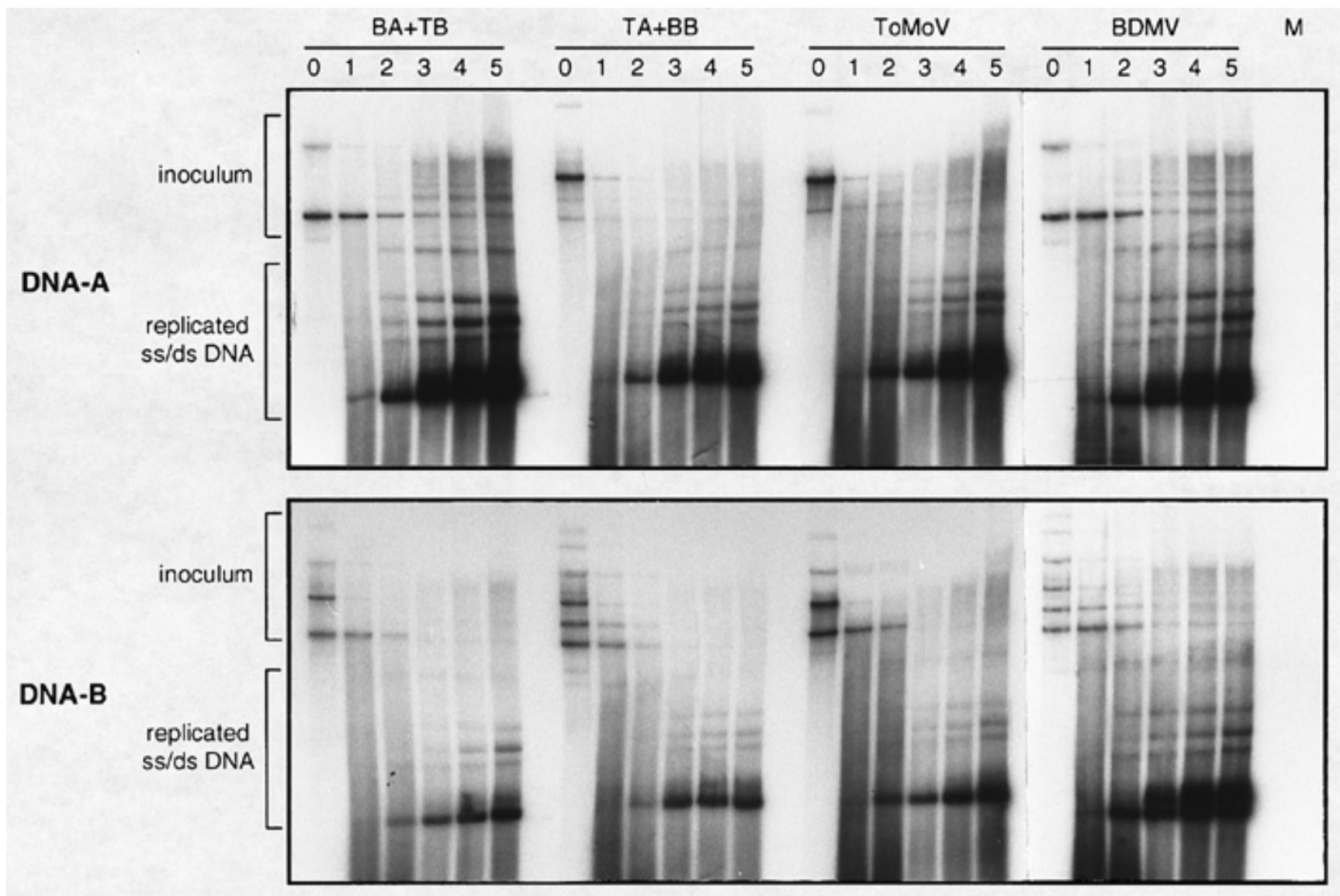

Fig. 5. Southern blot hybridization analysis of total nucleic acids extracted from Nicotiana tabacum Xanthi-nc cells transfected with BA+TB, $\mathrm{TA}+\mathrm{BB}$, ToMoV, and BDMV at 0, 24, 48, 72, 96, and $120 \mathrm{~h}$ post electroporation, lanes 0 to 5, respectively; and nontransfected cells (lane M). Blots were probed with specific probes for DNA-A or DNA-B. Positions of input inoculum and newly replicated viral DNA are indicated with brackets. 
The capsid protein, which has been shown to be required for long-distance movement of BGMV and TGMV in nonadapted hosts (Pooma et al. 1996; Jeffrey et al. 1996), may also be needed for BDMV and ToMoV to infect nonadapted hosts.

DNA-A infections have been reported in $N$. benthamiana plants agroinoculated with the DNA-A components of ACMV (Klinkenberg and Stanley 1990), Abutilon mosaic (Evans and Jeske 1993), and potato yellow mosaic geminiviruses (Buragohain et al. 1994). In the present study, plants having apparent DNA-A infections had been agroinoculated with different combinations of DNA-A and DNA-B and, thus, a transient contribution by the DNA-B component could not be ruled out (Petty et al. 1995). The establishment of symptomless DNA-A infections in plants agroinoculated with BDMV or ToMoV DNA-A provided direct evidence that DNA-B was not required for these infections. However, the activity of the BV1 and/or BC1 MPs, presumably at or near the site of inoculation, may facilitate DNA-A infections because higher infection rates and increased DNA-A levels were detected in plants agroinoculated with the pseudorecombinants compared with plants agroinoculated with DNA-A only. These results also establish that DNA-A infections are not unique to the permissive experimental host $N$. benthamiana, although higher infection rates and DNA-A levels were detected in $N$. benthamiana, compared with $P$. vulgaris or L. esculentum.

Based on a recent ultrastructural study of BDMV infection in $P$. vulgaris and $N$. benthamiana plants (Wang et al. 1996), a model for DNA-A infections mediated by agroinoculation can be proposed. DNA-A is released into phloem sieve elements at the point of agroinoculation from infected phloem parenchyma and/or companion cells. DNA-A, in the form of a virion or nucleoprotein complex, is then transported, long distance, via the phloem to the shoot apex, where infection occurs in protophloem cells. The symptomless phenotype of DNA-A infections likely reflects limited cell-to-cell movement in the absence of DNA-B-encoded MPs (Noueiry et al. 1994).

One of the characteristics of plants infected with the BDMV/ToMoV pseudorecombinants is reduced levels of DNA-B (Gilbertson et al. 1993a; Hou and Gilbertson 1996). The replication assays conducted in $N$. tabacum cells supported the hypothesis that DNA-B replication is less efficient for the pseudorecombinants. This is presumably due to the inefficient interaction of the Rep protein with the heterologous DNA-B CR. The overall differences in viral replication in the two cell lines reveal an influence of the host on the efficiency of viral replication, which may be due to differences in the function of certain viral and/or host factors. For example, greater replication of BDMV DNA-A versus ToMoV DNA-A in BY2 cells may reflect more efficient expression and/or function of the BDMV Rep protein. This would also account for the higher DNA-B levels detected for BA+TB versus $\mathrm{TA}+\mathrm{BB}$ in this cell line. Similarly, the higher levels of ToMoV DNA-A replication in Xanthi-nc versus BY2 cells may reflect more efficient expression and/or function of the ToMoV Rep protein. However, the reduced ToMoV DNA-B levels in Xanthi-nc cells suggest the role of additional factors in efficient ToMoV replication. Studies conducted on TGMV ACl repression in N. tabacum NT-1 and $N$. benthamiana cells revealed that AC1-mediated repression varied in these host species, and this was attributed to host factor differences (Eagle et al. 1994). The less efficient replication of ToMoV versus BDMV in N. tabacum may account for differences in infectivity of these viruses in $N$. tabacum plants. However, replication properties in $N$. tabacum cells are not necessarily an indicator of infectivity in other hosts because the more pathogenic pseudorecombinant, TA+BB, replicated less efficiently than BA+TB in $N$. tabacum BY2 cells.

In conclusion, the results of the present study establish that BDMV and ToMoV are adapted to P. vulgaris and L. esculentum, respectively. Furthermore, the infectivity of these two viruses and their pseudorecombinants in different hosts revealed the importance of the DNA-B component in host adaptation. Host adaptation probably involves efficient expression and/or function of the DNA-B-encoded MPs, and future studies should examine both of these possibilities.

\section{MATERIALS AND METHODS}

\section{Virus clones.}

Full-length infectious clones of BDMV (DNA-A in pBDA1 and DNA-B in pBDB1) and ToMoV (DNA-A monomer in pTFA-1, DNA-A dimer in pTFA-2, and DNA-B in pTFB-1) have been previously described (Gilbertson et al. 1991a, 1993a).

\section{Plasmid constructions.}

DNA manipulations were performed according to standard protocols (Sambrook et al. 1989; Ausubel et al. 1994). BDMV DNA-A and DNA-B and ToMoV DNA-B 1.5-mers were prepared as described by Paplomatas et al. (1994). For BDMV DNA-A, a 1.8-kb SacI/BglII fragment from pBDA1 was ligated into pSL1180 (Pharmacia, Piscataway, NJ) to generate pBDA-0.5. The full-length $B g l$ II DNA-A fragment from pBDA1 was ligated into pBDA- 0.5 to generate pBDA1.5. For BDMV DNA-B, a $1.1-\mathrm{kb} B g l \mathrm{II} /$ Bam HI fragment from $\mathrm{pBDB} 1$ was ligated into pSL1180 to generate pBDB0.5. The full-length BamHI DNA-B fragment from pBDB1 was ligated into $\mathrm{pBDB}-0.5$ to generate $\mathrm{pBDB}-1.5$. $\mathrm{pBDA}$ 1.5 and pBDB-1.5 were linearized with HindIII and KpnI, respectively, and individually ligated into the binary vector pCGN1547 (McBride and Summerfelt 1990) to generate pTBDA-1.5 and pTBDB-1.5. For ToMoV DNA-A, the HpaI/HindIII ToMoV DNA-A dimer fragment from pTFA-2 was ligated into pSL1180 to generate pTFA-2.1. The 5.2-kb BamHI/HindIII dimer fragment from pTFA-2.1 was ligated into pCGN1547 to generate pTTFA-2. For ToMoV DNA-B, a 1.2-kb EcoRI/ BamHI fragment from pTFB-1 was ligated into pKS (Stratagene, La Jolla, CA) to generate pTFB-0.5. The full-length BamHI DNA-B fragment from pTFB-1 was ligated into pTFB-0.5 to generate pTFB-1.5. pTFB-1.5 was linearized with $\mathrm{XbaI}$ and ligated into pCGN1547 to generate pTTFB-1.5.

Binary plasmids were individually transformed into Agrobacterium tumefaciens LBA4404 cells by a freeze/thaw method. Briefly, $1 \mu \mathrm{g}$ of DNA was added to $200 \mu \mathrm{l}$ of cells, and this mixture was quickly frozen in liquid $\mathrm{N}_{2}$ for $5 \mathrm{~min}$. The cells were thawed in a water bath at $37^{\circ} \mathrm{C}$ for $10 \mathrm{~min}$, incubated at $30^{\circ} \mathrm{C}$ for $4 \mathrm{~h}$, spread onto $2 \mathrm{XYT}$ plates supplemented with gentamicin $(10 \mu \mathrm{g} / \mathrm{ml})$, then incubated at $30^{\circ} \mathrm{C}$ for $48 \mathrm{~h}$. A. tumefaciens transformants were identified by PCR analysis. 


\section{Agroinoculation.}

Liquid cultures of A. tumefaciens were prepared in $2 \mathrm{XYT}$ supplemented with gentamicin $(10 \mu \mathrm{g} / \mathrm{ml})$. Cultures were adjusted to an OD of approximately 1.0 at $600 \mathrm{~nm}$. For inoculations that involved mixtures of viral components, the appropriate A. tumefaciens cultures were mixed in equal volumes. $L$. esculentum cv. Florida MH-1 (two-true-leaf stage), $N$. benthamiana (five- to seven-leaf stage), and N. tabacum cv. Xanthi-nc (seven- to 10-leaf stage) plants were agroinoculated by a stem puncture method. Briefly, the culture was taken up into a 1-ml syringe with a 27 -gauge needle, and the needle was pushed through the stem at three sites approximately 1 cm below the shoot apex. For $P$. vulgaris cv. Topcrop plants (primary leaf stage), the upper surface of one-half- to threequarters-expanded leaves was inoculated by a leaf scratch method. Inoculated plants were maintained in a growth chamber $\left(250 \mu \mathrm{E} \cdot \mathrm{s}^{-1} \cdot \mathrm{m}^{-2}\right.$ photosynthetically active radiation $16 \mathrm{~h}$ per day, $30^{\circ} \mathrm{C}$ ), and symptoms were evaluated 14 to 21 days after inoculation.

\section{Protoplast replication assay.}

Protoplasts were prepared from N. tabacum BY2 and Xanthi-nc suspension cell cultures as described by Fromm et al. (1987). Cells were digested at room temperature with $1 \%$ (wt/vol) cellulysin (Calbiochem, La Jolla, CA), 0.6\% (wt/vol) cellulase RS (Yakult, Tokyo), 0.4\% (wt/vol) macerase (Yakult), $5 \mathrm{mM} \mathrm{CaCl}_{2}, 12 \mathrm{mM}$ sodium acetate, and $0.45 \mathrm{M}$ mannitol for 3 to $5 \mathrm{~h}$. Protoplasts were washed twice in $5 \mathrm{mM}$ $\mathrm{CaCl}_{2}, 12 \mathrm{mM}$ sodium acetate, and $0.45 \mathrm{M}$ mannitol, and resuspended in $10 \mathrm{mM}$ HEPES ( $N$-2-hydroxyethylpiperazine$N^{\prime}$-2-ethanesulfonic acid), $10 \mathrm{mM} \mathrm{NaCl}, 10 \mathrm{mM} \mathrm{KCl}, 4 \mathrm{mM}$ $\mathrm{CaCl}_{2}$, and $0.2 \mathrm{M}$ mannitol. For each virus, $10 \mu \mathrm{g}$ each of DNA-A and DNA-B (monomers or 1.5-mers/dimers) was electroporated into approximately $2 \times 10^{6}$ protoplasts in a volume of $0.7 \mathrm{ml}$ with a PG200 Progenitor II (Hoefer, San Francisco) at $290 \mathrm{~V}$ and $490 \mu \mathrm{F}$ for $8 \mathrm{~ms}$. The electroporated protoplasts were kept on ice for $10 \mathrm{~min}$, then diluted with $9 \mathrm{ml}$ of growth medium $(5 \%$ [vol/vol] coconut water and $0.2 \mathrm{M}$ mannitol in KCMS medium [Murashige Minimal Organics Medium; Gibco BRL, Grand Island, NY]) and maintained at room temperature in the dark. Approximately $2 \times 10^{5}$ cells were removed at $0,24,48,72,96$, and 120 hpe for DNA extraction. Cells were homogenized in STE solution $(50 \mathrm{mM}$ Tris $\mathrm{pH} 8.0,100 \mathrm{mM} \mathrm{NaCl}$, and $10 \mathrm{mM}$ EDTA) in a tissue grinder, and extracted twice with an equal volume of phenol/chloroform (1:1). Total nucleic acids were precipitated with ethanol, recovered by centrifugation $(10,000 \times g$ for 10 $\mathrm{min}$ ), and resuspended in Tris-EDTA (TE) buffer.

\section{Detection of geminivirus DNA \\ by Southern blot hybridization and PCR analyses.}

The second youngest leaves were collected, and total nucleic acids were extracted as described by Hou and Gilbertson (1996). Approximately 0.5 or $2.0 \mu \mathrm{g}$ of total nucleic acids from symptomatic and symptomless plants, respectively, and approximately $1 \mu \mathrm{g}$ of total nucleic acids from transfected $N$. tabacum cells were fractionated in $1.2 \%$ agarose gels in Trisacetate-EDTA buffer and transferred to Hybond $\mathrm{N}^{+}$membranes (Amersham, Arlington Heights, IL). Blots were hybridized separately with BDMV/ToMoV DNA-A-specific and DNA-B-specific probes (Hou and Gilbertson 1996) according to methods described in Sambrook et al. (1989). The specific activity of each probe was determined with a scintillation counter, and similar amounts of probe $(\mathrm{cpm} / \mathrm{ml})$ were used in each hybridization reaction. Probes were removed from blots by washing in $0.4 \mathrm{~N} \mathrm{NaOH}$ for $30 \mathrm{~min}$ at $25^{\circ} \mathrm{C}$, then in $0.1 \times$ SSC ( $1 \times \mathrm{SSC}$ is $0.15 \mathrm{M} \mathrm{NaCl}$ plus $0.015 \mathrm{M}$ sodium citrate), $0.1 \%$ sodium dodecyl sulfate (SDS), and 0.2 M Tris (pH 7.5) for $15 \mathrm{~min}$ at $45^{\circ} \mathrm{C}$. Fifty nanograms of linear BDMV and ToMoV DNA-A and DNA-B was included in each gel to confirm that similar hybridization signals were obtained for each probe, and direct comparisons were made between blots having equivalent hybridization signals.

Viral DNA levels were estimated from autoradiographs by densitometry (IS-1000 Digital Imagining System, Alpha Innotech Corporation, San Leandro, CA). To compensate for the overexposure of certain treatments, autoradiographs having shorter exposures were used to determine the appropriate area to be scanned for quantification. For infected plants, viral DNA levels are presented as means of two to four inoculated plants. For protoplast assays, average replication levels for each DNA component were determined for the 120 hpe time point for three independent experiments in which 1.5-mers/ dimers were used as inoculum.

DNA extraction for PCR analysis, degenerate DNA-A primers (PAL1v1978 and PAR1c475) and DNA-B primers (PBL1v2040 and PBV1c703) for detection of whiteflytransmitted geminiviruses, and PCR conditions have been previously described (Gilbertson et al. 1991b; Rojas et al. 1993; Hou and Gilbertson 1996).

\section{ACKNOWLEDGMENTS}

We thank Mysore R. Sudarshana and Maria R. Rojas for critical reading of the manuscript, Pablo Guzman for technical assistance, and Jeff Hall for assistance in preparation of figures. This work was supported in part by fellowships from the College of Agricultural and Environmental Sciences, University of California-Davis to Y.-M. H., and by grants from Seminis Vegetable Seed Company and the United States Department of Agriculture National Research Initiative Competitive Grants Program (9301256) to R. L. G.

\section{LITERATURE CITED}

Abouzid, A. M., Polston, J. E., and Hiebert, E. 1992. The nucleotide sequence of tomato mottle virus, a new geminivirus isolated from tomatoes in Florida. J. Gen. Virol. 73:3225-3229.

Arguello-Astorga, G. R., Guevara-Gonzalez, R. G., Herrera-Estrella, L. R., and Rivera-Bustamante, R. F. 1994. Geminivirus replication origins have a group-specific organization of iterative elements: A model for replication. Virology 203:90-100.

Ausubel, F. M., Brent, R., Kingston, R. E., Moore, D. D., Seidman, J. G., Smith, J. A., and Struhl, K. 1994. Current Protocols in Molecular Biology. John Wiley \& Sons, New York.

Briddon, R. W., and Markham, P. G. 1995. Geminiviridae. Pages 158165 in: Virus Taxonomy: Classification and Nomenclature of Viruses. F. A. Murphy, C. M. Fauquet, D. H. L. Bishop, S. A. Ghabrial, A. W. Jarvis, G. P. Martelli, M. A. Mayo, and M. D. Summers, eds. Springer-Verlag, New York.

Buragohain, A. K., Sung, Y. K., Coffin, R. S., and Coutts, R. H. A. 1994. The infectivity of dimeric potato yellow mosaic geminivirus clones in different hosts. J. Gen. Virol. 75:2857-2861.

Dawson, W. O., and Hilf, M. E. 1992. Host-range determinants of plant viruses. Annu. Rev. Plant Physiol. Plant Mol. Biol. 43:527-555.

Eagle, P. A., Orozco, B. M., and Hanley-Bowdoin, L. 1994. A DNA sequence required for geminivirus replication also mediates transcriptional regulation. Plant Cell 6:1157-1170. 
Evans, D., and Jeske, H. 1993. DNA B facilitates, but is not essential for, the spread of Abutilon mosaic virus in agroinoculated Nicotiana benthamiana. Virology 194:752-757.

Faria, J. C., Gilbertson, R. L., Hanson, S. F., Morales, F. J., Ahlquist, P., Loniello, A. O., and Maxwell, D. P. 1994. Bean golden mosaic geminivirus type II isolates from the Dominican Republic and Guatemala: Nucleotide sequences, infectious pseudorecombinants, and phylogenetic relationships. Phytopathology 84:321-329.

Frischmuth, T., Roberts, S., von Arnim, A., and Stanley, J. 1993. Specificity of bipartite geminivirus movement proteins. Virology 196:666673.

Fromm, M., Callis, J., Taylor, L. P., and Walbot, V. 1987. Electroporation of DNA and RNA into plant protoplasts. Methods Enzymol. 153:351-365.

Gilbertson, R. L., Faria, J. C., Hanson, S. F., Morales, F. J., Ahlquist, P., Maxwell, D. P., and Russell, D. R. 1991a. Cloning of the complete DNA genomes of four bean-infecting geminiviruses and determining their infectivity by electric discharge particle acceleration. Phytopathology 81:980-985.

Gilbertson, R. L., Hidayat, S. H., Paplomatas, E. J., Rojas, M. R., Hou, Y.-M., and Maxwell, D. P. 1993a. Pseudorecombination between infectious cloned DNA components of tomato mottle and bean dwarf mosaic geminiviruses. J. Gen. Virol. 74:23-31.

Gilbertson, R. L., Paplomatas, E. J., Grieco, P. D., Patel, V. P., and Maxwell, D. P. 1993b. Agroinfection of bean, tobacco, and tomato with two bipartite geminiviruses isolated from tomato. Phytopathology 83:1350.

Gilbertson, R. L., Rojas, M. R., Russell, D. R., and Maxwell, D. P. $1991 \mathrm{~b}$. Use of the asymmetric polymerase chain reaction and DNA sequencing to determine genetic variability of bean golden mosaic geminivirus in the Dominican Republic. J. Gen. Virol. 72:2843-2848.

Haley, A., Richardson, K., Zhan, X., and Morris, B. 1995. Mutagenesis of the $\mathrm{BC} 1$ and $\mathrm{BV} 1$ genes of African cassava mosaic virus identifies conserved amino acids that are essential for spread. J. Gen. Virol. 76: 1292-1298.

Hofer, P., Engel, M., Jeske, H., and Frischmuth, T. 1997. Nucleotide sequence of a new bipartite geminivirus isolated from the common weed Sida rhombifolia in Costa Rica. J. Gen. Virol. 78:1785-1790.

Hou, Y.-M., and Gilbertson, R. L. 1996. Increased pathogenicity in a pseudorecombinant bipartite geminivirus correlates with intermolecular recombination. J. Virol. 70:5430-5436.

Ingham, D. J., and Lazarowitz, S. G. 1993. A single missense mutation in the BR1 movement protein alters the host range of the squash leaf curl geminivirus. Virology 196:694-702.

Ingham, D. J., Pascal, E., and Lazarowitz, S. G. 1995. Both bipartite geminivirus movement proteins define viral host range, but only BL1 determines viral pathogenicity. Virology 207:191-204.

Jeffrey, J. L., Pooma, W., and Petty, I. T. D. 1996. Genetic requirements for local and systemic movement of tomato golden mosaic virus in infected plants. Virology 223:208-218.

Klinkenberg, F. A., and Stanley, J. 1990. Encapsidation and spread of African cassava mosaic virus DNA A in the absence of DNA B when agroinoculated to Nicotiana benthamiana. J. Gen. Virol. 71:1409-1412.

Laufs, J., Jupin, I., Davis, C., Schumacher, S., Heyraud-Nitschke, F., and Gronenborn, B. 1995. Geminivirus replication: Genetic and biochemical characterization of Rep protein function, a review. Bio- chimie 77:765-773

Lazarowitz, S. G. 1991. Molecular characterization of two bipartite geminiviruses causing squash leaf curl disease: Role of viral replication and movement functions in determining host range. Virology 180:70-80.

McBride, K. E., and Summerfelt, K. R. 1990. Improved binary vectors for Agrobacterium-mediated plant transformation. Plant Mol. Biol. 14:269-276.

Mise, K., and Ahlquist, P. 1995. Host-specificity restriction by bromovirus cell-to-cell movement protein occurs after initial cell-to-cell spread of infection in nonhost plants. Virology 206:276-286.

Morales, F., Niessen, A., Ramírez, B., and Castaño, M. 1990. Isolation and partial characterization of a geminivirus causing bean dwarf mosaic. Phytopathology 80:96-101.

Noueiry, A. O., Lucas, W. J., and Gilbertson, R. L. 1994. Two proteins of a plant DNA virus coordinate nuclear and plasmodesmal transport. Cell 76:925-932.

Padidam, M., Beachy, R. N., and Fauquet, C. M. 1995. Classification and identification of geminiviruses using sequence comparisons. J. Gen. Virol. 76:249-263.

Paplomatas, E. J., Patel, V. P., Hou, Y.-M., Noueiry, A. O., and Gilbertson, R. L. 1994. Molecular characterization of a new sap-transmissible bipartite genome geminivirus infecting tomatoes in Mexico. Phytopathology 84:1215-1224.

Pascal, E., Goodlove, P. E., Wu, L. C., and Lazarowitz, S. G. 1993 Transgenic tobacco plants expressing the geminivirus BL1 protein exhibit symptoms of viral disease. Plant Cell 5:795-807.

Petty, I. T. D., Miller, C. G., Meade-Hash, T. J., and Schaffer, R. L. 1995. Complementable and noncomplementable host adaptation defects in bipartite geminiviruses. Virology 212:263-267.

Pooma, W., Gillette, W. K., Jeffrey, J. L., and Petty, I. T. D. 1996. Host and viral factors determine the dispensability of coat protein for bipartite geminivirus systemic movement. Virology 218:264-268.

Rojas, M. R., Gilbertson, R. L., Russell, D. R., and Maxwell, D. P. 1993. Use of degenerate primers in the polymerase chain reaction to detect whitefly-transmitted geminiviruses. Plant Dis. 77:340-347.

Rybicki, E. P. 1994. A phylogenetic and evolutionary justification for three genera of Geminiviridae. Arch. Virol. 139:48-78.

Sambrook, J., Fritsch, E. F., and Maniatis, T. A. 1989. Molecular Cloning: A Laboratory Manual. 2nd ed. Cold Spring Harbor Laboratory, Cold Spring Harbor, NY.

Schaffer, R. L., Miller, C. G., and Petty, I. T. D. 1995. Virus and hostspecific adaptations in the $B L 1$ and $B R 1$ genes of bipartite geminiviruses. Virology 214:330-338.

Stenger, D. C., Davis, K. R., and Bisaro, D. M. 1992. Limited replication of tomato golden mosaic virus DNA in explants of nonhost species. Mol. Plant-Microbe Interact. 5:525-527.

Timmermans, M. C. P., Das, O. P., and Messing, J. 1994. Geminiviruses and their uses as extrachromosomal replicons. 1994. Annu. Rev. Plant Physiol. Plant Mol. Biol. 45:79-112.

von Arnim, A., and Stanley, J. 1992. Determinants of tomato golden mosaic virus symptom development located on DNA B. Virology 186:286-293

Wang, H. L., Gilbertson, R. L., and Lucas, W. J. 1996. Spatial and temporal distribution of bean dwarf mosaic geminivirus in Phaseolus vulgaris and Nicotiana benthamiana. Phytopathology 86:1204-1214. 